\title{
1 The sources of sex differences in aging in annual fishes
}

3 Martin Reichard ${ }^{\mathrm{a}, \mathrm{b}}$, Radim Blažek $^{\mathrm{a}}$, Jakub Žák ${ }^{\mathrm{a}, \mathrm{c}}$, Petr Kačer $^{\mathrm{d}}$, Oldřich Tomášek ${ }^{\mathrm{a}, \mathrm{c}}$, Tomáš Albrechta, $^{\mathrm{a}, \mathrm{c}}$,

$4 \quad$ Alessandro Cellerino ${ }^{\mathrm{e}, \mathrm{f}} \&$ Matej Polačik ${ }^{\mathrm{a}}$

$6 \quad{ }^{a}$ Czech Academy of Sciences, Institute of Vertebrate Biology, Květná 8, 603 65, Brno, Czech

$7 \quad$ Republic

$8 \quad{ }^{b}$ Department of Botany and Zoology, Faculty of Science, Masaryk University, Kotlářská 2, 61137

9 Brno, Czech Republic

$10{ }^{\mathrm{c}}$ Department of Zoology, Faculty of Science, Charles University, Viničná 7, 12800 Prague, Czech

11 Republic

12 d Czech University of Life Sciences, Kamýcká 129, 16500 Prague 6, Czech Republic

13 e Bio@SNS, Scuola Normale Superiore, Department of Neurosciences, Piazza dei Cavalieri 7,

1456126 Pisa, Italy

15 f Fritz Lipmann Institute for Age Research, Leibniz Institute, Beutenbergstr. 11, D-07745, Jena,

16 Germany

18 ORCID; MR: 0000-0002-9306-0074; RB: 0000-0003-1150-0273; JŽ: 0000-0003-2845-8323; OT:

19 0000-0002-2657-5916; TA: 0000-0002-9213-0034; AC: 0000-0003-3834-0097; MP: 0000-0003-

$20 \quad 1309-2146$

22 Corresponding author: Martin Reichard, Institute of Vertebrate Biology, Czech Academy of

23 Sciences, Květná 8, 60365 Brno, Czech Republic; telephone: +420543422522, email:

24 reichard@ivb.cz 


\section{Abstract}

26 Sex differences in lifespan and aging are widespread among animals, with males usually the

27 shorter-lived sex. Despite extensive research interest, it is unclear how lifespan differences between

28 the sexes are modulated by genetic, environmental and social factors. We combined comparative

29 data from natural populations of annual killifishes with experimental results on replicated captive

30 populations, showing that females consistently outlived males in the wild. This sex-specific survival

31 difference persisted in social environment only in two most aggressive species, and ceased

32 completely when social and physical contacts were prevented. Demographically, neither an earlier

33 start nor faster rate of aging accounted for shorter male lifespans, but increased baseline mortality

34 and the lack of mortality deceleration in the oldest age shortened male lifespan. The sexes did not

35 differ in any measure of functional aging we recorded. Overall, we demonstrate that sex differences

36 in lifespan and aging may be ameliorated by modulating social and environmental conditions. 


\section{Introduction}

38 Males and females differ in many demographic and life history parameters, with important

39 consequences for species ecology, evolution, and physiology, as well as for practical and societal

40 outcomes (Trivers 1972, Austad 2006, Regan and Partridge 2013). Inter-sexual differences in

41 lifespan (age at death) and aging (increase in mortality risk associated with deterioration in bodily

42 functions) are widespread among animals, from nematodes to humans (Austad and Fischer 2016).

43 Males are usually the shorter-lived sex (Promislow 2003; Liker and Szekély 2005, Lemaitre et al.

44 2020), but there is substantial unexplained variation among species and populations (Austad and

45 Fischer 2016). Inter-sexual differences in lifespan and aging appear modulated by environmental

46 and social factors (Austad 2006, Lemaitre et al. 2020), but their effects remain opaque.

Why do males typically express a truncated lifespan in comparison with females? One set of explanations posits that the primary difference stems from genetic and genomic differences between

the sexes (Gemmel et al. 2004; Maklakov and Lummaa 2013). In mammals, fruit flies and many

other taxa, males are the heterogametic sex and hemizygosity of key genes located on the sex

51 chromosomes resulting in an inability to compensate for the effects of deleterious mutations have

52 been implicated in shorter male lifespan (Trivers 1985; Xirocostas et al. 2020). However, males

53 also show shorter lifespans in many birds and butterflies, despite female heterogamy in those

54 groups (Gotthard et al. 2000; Tompkins and Anderson 2019; Sielezniew et al. 2020). Asymmetry in

55 the inheritance of mitochondria, leading to suboptimal compatibility between mitochondrial and

56 nuclear genomes in males (Frank and Hurst 1996), could explain male-biased mortality and aging in

57 heterogametic taxa (Gemmel et al. 2004). Yet these explanations cannot account for the large

58 variation in the sex bias in lifespan and aging rate seen within species and among inbred laboratory 
strains housed under contrasting conditions (Austad and Fisher 2016), strongly implicating other

60 factors in driving sex-biased mortality.

Males and females differ in their routes to reproductive success (Trivers 1972). These

62 divergent trajectories arise as a consequence of gamete size disparity which leads to variation in

63 reproductive roles of males and females. This disparity is best explained by sexual selection, with

64 asymmetric variation in reproductive success between the sexes (Andersson 1994). Male

65 reproductive success is often skewed towards a few highly successful individuals while female

66 reproductive success is far less variable (Arnold 1994). Mating system is a key modulator of this

67 variation. In a monogamous mating system, differences in the variance in reproductive success

68 between males and females are trivial. In highly polygynous mating systems, such as those with

69 male harems, a single male may monopolize a large number of females generating highly skewed

70 male reproductive success (Clutton-Brock and Isvaran 2007).

71 Sexual selection is associated with elevated mortality in the more competitive sex (Székely

72 et al. 2014). Conspicuous signaling to rivals and potential partners directly increases the risk of

73 mortality from predators (Tuttle and Ryan 1991). Male-male competition is also risky and may lead

74 to increased mortality (Beirne et al. 2015). Higher male mortality is often precipitated via

75 alterations to hormonal profiles, resulting in chronic stress (Keller et al. 1992) or elevated

76 testosterone levels (Foo et al. 2017) thereby making individuals more susceptible to infections or

77 physiological deterioration (Moore and Wilson 2002, Gupta et al. 2020).

African annual fishes from the genus Nothobranchius are an ideally suited model taxon for

79 biomedical and evolutionary questions related to aging (Cellerino et al. 2016, Hu and Brunet 2018,

80 Cui et al. 2019). Inhabiting ephemeral savanna pools, they have evolved naturally short lifespans

81 which recapitulate typical features of vertebrate aging, including multifarious functional 
82 deterioration in old age (Cellerino et al. 2016; Hu and Brunet 2018). In the wild, killifish hatch at

83 the onset of the rainy season from desiccation-resistant eggs. Both sexes grow rapidly and achieve

84 sexual maturity in as few as two weeks (Vrtílek et al. 2018a). Males compete for access to females,

85 with a marked variability in the strength of intra-sexual competition among species (Wildekamp

86 2004, Genade 2005, Polačik and Reichard 2011; Cellerino et al. 2016). Natural lifespan is limited

87 by desiccation of their habitat, but most fish succumb long before their natal pool desiccates

88 (Vrtílek et al. 2018b). Strikingly, a short lifespan of several months is retained in captivity, where

89 fish are shielded from extrinsic mortality, with captive fish suffering a range of functional declines

90 (Cellerino et al. 2016). In all Nothobranchius species for which information on sex chromosomes is

91 available, males are the heterogametic sex, though sex chromosomes are rarely morphologically

92 distinguishable (Krysanov and Demidova 2018).

93 We combined data from wild populations with experimental results from captive fish to

94 disentangle the causes of differences in lifespan and aging between male and female African annual

95 killifish. Using a set of four species (each replicated as two independent populations), we compared

96 demographic and functional aging between the sexes. Overall, we demonstrate that sex differences

97 in lifespan and aging are primarily modulated by social and environmental conditions.

99 Results

100 Sex ratio in wild and captive populations. Using adult sex ratios from 376 wild populations

101 (15,968 fish), we found that natural killifish populations in three study species were sex-biased,

102 with significantly more females. Sex ratios in one species (N. kadleci) were equal (Fig. 1). This

103 finding corroborated the outcomes of a previous study (Reichard et al. 2014), which was reinforced

104 here using a larger dataset. 
106 results could result from the cumulative effects of biases in primary sex ratios and sex-dependent

107 mortality and to quantify sex-specific survival, an estimate of sex ratios at the onset of adulthood is

108 needed. To obtain these data we raised 63 cohorts of outbred, wild-derived captive populations

109 from study species in protected laboratory conditions. We found that sex ratios in protected

110 conditions were equal in the three study species that exhibited female-biased sex ratio in the wild,

111 while the sex ratio was male-biased in $N$. kadleci - the species with an equal sex ratio in the wild

112 (Fig. 1). This finding implies that mortality of adult males in natural populations was consistently

113 higher than female mortality in all four species.

115 Sex differences in lifespan - social and environmental effects. To investigate proximate causes

116 of sex-biased mortality, we raised a set of killifish cohorts from a total of 8 wild-derived

117 populations from all 4 species (Supplementary Table 1) in the laboratory and compared sex

118 differences in lifespan and aging in two contrasting social treatments. By using captive breeding we

119 excluded predation (and predation risk), which has been implicated in sex-biased mortality in wild

120 fish and other animals (Székely et al. 2014), from both treatments. The first treatment comprised

121 replicated social groups of 10-12 fish (equal sex ratio) in which males and females interacted freely,

122 competed and formed dominance hierarchies ( $\mathrm{N}=84$ groups). The second treatment comprised

123 singly-housed fish $(\mathrm{N}=178$ fish). We predicted that in a captive setting sex differences in mortality

124 would be removed if predation is the source of male-biased mortality. If social stress elevates male

125 mortality, we predicted persistence of male-biased mortality in the social treatment but its

126 disappearance in singly-housed fish treatment. Finally, if intrinsic, sex-specific functional 
127 deterioration causes male-biased mortality, we predicted male-biased mortality to persists in both 128 captive treatments.

We found support for predation-related and social stress-related decreases in male lifespan.

130 First, sex differences in lifespan in social tanks persisted in two species $-N$. orthonotus $(\mathrm{z}=4.84, \mathrm{P}$

$131<0.001$; with male median lifespan $42 \%$, i.e. 76 days shorter $)$ and $N$. furzeri $(\mathrm{z}=2.64, \mathrm{P}=0.008$;

132 male lifespan $24 \%$, i.e. 29 days shorter $)$ but disappeared in the other two $-N$. kadleci $(\mathrm{z}=0.24, \mathrm{P}=$ $1330.81)$ and $N$.pienaari $(\mathrm{z}=0.26, \mathrm{P}=0.79$; Table 1$)$. This demonstrates that the absence of predation

134 eliminated the sex bias in mortality in two species, but not in the other two. This interspecific

135 variation in socially-induced sex bias in lifespan tightly covaries with the level of male

136 aggressiveness, which is markedly higher in N. orthonotus, followed by N.furzeri, N. kadleci and

137 N.pienaari (Wildekamp 2004, Genade 2005, Polačik and Reichard 2011).

138 Second, there was no sex bias in lifespan when fish were housed singly (N. orthonotus: $\mathrm{z}=$

$1390.14, \mathrm{P}=0.89 ;$ N. furzeri: $\mathrm{z}=0.50, \mathrm{P}=0.62 ;$ N. kadleci: $\mathrm{z}=1.28, \mathrm{P}=0.20 ; N$. pienaari: $\mathrm{z}=1.54, \mathrm{P}$

$140=0.12$ ). Lifespan estimates of fish that lived in social and singly-housed treatments were congruent

141 (95\% confidence intervals for median lifespans overlapped) except for an increase in median

142 lifespan in singly-housed $N$. orthonotus males (Table 1), the most aggressive species. This finding

143 implies that male-male aggression considerably decreased male lifespan in N. orthonotus in a social

144 setting.

145 Finally, we tested the hypothesis that the sex bias in mortality observed in challenging

146 natural conditions disappeared in more benign conditions in captivity, using one population of $N$.

147 furzeri. We replicated a strong circadian fluctuation in water temperature (from $20 \pm 1^{\circ} \mathrm{C}$ in early

148 morning to $35 \pm 1^{\circ} \mathrm{C}$ in late afternoon), which is characteristic of the natural environment (Žák et al.

1492018 ) and exceeds killifish preferred temperature variation by $6^{\circ} \mathrm{C}$ (Polačik et al. 2016, Žák et al. 
2018). This thermal challenge is not being employed in standard breeding protocol for captive

151 killifish (Polačik et al. 2016), which we imposed in our main experiment. We found that even under

152 these challenging environmental conditions, there was no sex bias in mortality in singly-housed $N$.

153 furzeri (thermally fluctuating environment: $\mathrm{z}=0.55, \mathrm{P}=0.582, \mathrm{~N}=45 \mathrm{~N}$. furzeri kept as singly-

154 housed fish; control stable temperature of $27.5 \pm 1^{\circ} \mathrm{C}: \mathrm{z}=1.04, \mathrm{P}=0.297, \mathrm{~N}=45$ ).

Sex differences in actuarial aging. Sex-biased lifespan can arise from differences in baseline mortality (i.e. one sex experiencing persistently higher mortality) or demographic rate of aging (i.e. a steeper increase in mortality with age in one sex). These two sources of differential mortality can be best estimated with a Gompertz model of increasing failure time (Bronikowski et al. 2011;

160 Boonekamp et al. 2020). We fitted a set of models (Colchero et al. 2012) to our data from the social

161 treatment, in which shorter male lifespan was detected, and confirmed that Gompertz-family

162 models well approximated observed mortality patterns (Supplementary Table 2). We used Bayesian 163 survival trajectory analysis (BaSTA; Colchero et al. 2012) to estimate intersexual differences in 164 actuarial ageing within populations of species with sex-biased mortality. In $N$. orthonotus, the species with the greatest contrast in lifespan between the sexes, male-

166 biased mortality was affected by stronger baseline mortality in males and not their higher rate of 167 aging, consistently across both study populations (Figure 2a, b). In N.furzeri, the second species 168 with male-biased mortality in the social treatment, a Gompertz-logistic model (which includes an 169 additional parameter describing deceleration in the aging rate in old age) gave a significantly better 170 fit to observed data than a simple Gompertz model (Supplementary Table 2). We detected lower 171 mortality deceleration in old age (parameter $c$ ) in one $N$. furzeri population (Figure 2c) and no 172 intersexual difference in Gompertz model parameters in the second population (Figure 2d). 
174 Sex differences in functional aging. In a protected environment, mortality derives from

175 deterioration in bodily function. We contrasted data on biomarkers of cellular and physiological

176 aging between males and females kept in the social treatment. We analyzed markers of oxidative

177 stress to lipids, proteins and DNA in liver, brain and heart tissues of young (14 weeks) and old (24

178 weeks) fish from all 8 experimental populations using liquid chromatography-electrospray-high

179 resolution mass spectrometry. A PCA-based composite value well approximated oxidative stress

180 across tissues and markers (PC1 explained 72\% of variation, Supplementary Table 3). Oxidative

181 stress increased with age (LMM: $\left.\mathrm{t}_{140}=24.89, \mathrm{P}<0.001\right)$ but did not differ between the sexes in

182 either absolute values $\left(\mathrm{t}_{140}=0.14, \mathrm{P}=0.886\right)$ nor in the steepness of its increase with age (sex by

183 age interaction: $\left.\mathrm{t}_{140}=0.49, \mathrm{P}=0.629\right)$. The same outcome was obtained from species-specific

184 analyses (Supplementary Table 4) and for biomarker- and tissue-specific analyses (Supplementary

185 Table 5). Hence, there was no detectable intersexual difference in oxidative stress.

186 Using a different set of individuals, we compared the deposition of lipofuscin in liver tissue,

187 as a biomarker of cellular aging. Lipofuscin is an aggregate of oxidized proteins that accumulates in

188 aged post-mitotic cells (Jung et al. 2006). Lipofuscin deposition did not differ between males and

189 females (LMM with Poisson error distribution: $\mathrm{z}=0.17, \mathrm{P}=0.862, \mathrm{~N}=75$ fish) and we found no

190 significant increase in lipofuscin accumulation with age $(\mathrm{z}=1.57, \mathrm{P}=0.115$; sex by age interaction:

$191 \mathrm{z}=0.65, \mathrm{P}=0.514)$. Finally, as Nothobranchius killifish age they suffer proliferative changes

192 leading to organ dysfunction that is linked to mortality and which can be revealed by

193 histopathological examination (di Cicco et al. 2010, Baumgart et al. 2015). We found no intersexual

194 differences in proliferative changes in the kidney $(\mathrm{P}=0.847)$ or liver $(\mathrm{P}=0.115$; Table 2$)$. 


\section{Discussion}

197 Intersexual differences in lifespan and aging are widespread among taxa, but despite a substantial

198 research interest, it is still not clear how genetic differences between the sexes are modulated by

199 environmental and social factors (Austad 2006; Gordon et al. 2017; Lemaitre et al. 2020). Using

200 eight populations from four closely related annual killifish species, we combined comparative and

201 experimental approaches to demonstrate that female bias in wild annual killifish populations arises

202 from a combination of higher extrinsic male mortality in natural populations and higher intrinsic

203 mortality linked to social interactions, rather than from generalized intersexual differences in

204 functional deterioration. Females consistently outlived males in the wild, but this difference

205 persisted in social tanks only in more aggressive species, and ceased when fish were housed singly.

206 Increased baseline mortality, not an earlier or faster rate of aging was primarily responsible for a

207 shorter male lifespan in a social setting. Importantly, there were no differences between the sexes in

208 a series of measures of functional aging (oxidative stress, lipofuscin deposition, or age-related

209 proliferative changes in liver and kidney).

210 The impacts of sexual selection explained male-biased mortality in natural and experimental

211 annual killifish populations. In the wild, there is evidence that males suffer elevated predation.

212 Annual killifish are highly sexually dichromatic, with brightly colored males and dull females

213 (Sedláček et al. 2014), and visually hunting birds, such as herons and kingfishers, are the main

214 predators of annual killifish (Haas, 1976, Keppeler et al. 2016; Reichard and Polačik 2019). A

215 mortality cost of showy sexually-selected traits is a well-recognized source of intersexual

216 differences in lifespan (Promislow et al. 1992; Székely et al. 2014, Lemaitre et al. 2020). In

217 addition, we found male-male competition for mating opportunities significantly contributed to

218 elevated male mortality in more aggressive species. Notably, we observed male combat-related 
219 injuries in five $N$. orthonotus and three $N$. furzeri males that died in the social group treatment.

220 Persistent stress (Keller et al. 1992), possibly mediated by elevated levels of corticosteroids (Foo et

221 al. 2017), is frequently associated with increased mortality (Moore and Wilson 2002), but hormonal

222 profiles were not measured in our study. Unexpectedly, we detected no functional characteristics

223 underlying a higher male baseline mortality in our study, despite using measures of physiological

224 aging that were previously found to be suitable biomarkers of functional decline as they predictably

225 varied with age and among killifish species and populations (Terzibasi-Tozzini et al. 2013;

226 Baumgart et al. 2015; Blažek et al. 2017). This is comparable to well-described male-female health-

227 survival paradox in humans, where woman outlive men despite experiencing greater levels of

228 functional problems at older age (reviewed in Gordon et al. 2017).

229 One species (N. kadleci) exhibited equal adult sex ratios in the wild and a male-biased sex

230 ratio at sexual maturity in captivity, consistently across populations and cohorts. Sowersby et al.

231 (2020) hypothesized that sex ratios can evolve extremely rapidly in killifish, demonstrating large

232 interspecific differences among adult sex ratios across 15 annual and non-annual killifish species

233 raised in the lab. We propose that male-biased sex ratio in $N$. kadleci might have evolved as

234 compensatory mechanism to mitigate male-biased mortality in natural populations. Some wild

235 populations in our dataset presented extremely female-skewed sex ratios (e.g. in one natural

236 population we have collected one male and 44 females), and production of male-biased progeny

237 would be adaptive in such populations in accordance with Fisher's principle (Fisher 1930). Ongoing

238 cyto(genetic) research aims to characterize the nature of this potential compensatory mechanism.

Despite an enormous research effort, a comprehensive causal understanding of sex

240 differences in lifespan and aging remains elusive, probably because it comprises a series of complex

241 underlying sources. Mammals are arguably the best studied vertebrate taxon with respect to aging. 
242 A recent comparative study that combined data from 101 mammalian species demonstrated that

243 females lived on average 19\% longer than conspecific males but without finding any consistent

244 intersexual differences in aging rates (Lemaitre et al.2020), in line with our experimental results

245 with killifishes. The fact that heterospecific-sex disadvantage is much stronger in male

246 heterogametic systems (21\% longer lifespan of homogametic females) than female heterogametic

247 systems (7\% longer lifespan of homogametic males) (Xirocostas et al. 2020) highlights the

248 importance of reproductive roles and mating systems in shaping intersexual lifespan differences.

249 Here, we have demonstrated that sexual selection, which acts differently on the sexes, substantially

250 alters sex differences in lifespan and aging through multiple processes even within an ecologically

251 and evolutionary discrete lineage, and that these effects are strongly moderated by the social and

252 environmental setting. 


\section{Materials and Methods}

254 Sex ratio estimates from wild populations. We estimated the sex ratio in wild populations of all

255 four study species from 10 field trips to Mozambique conducted between 2008 and 2015.

256 Nothobranchius populations contain a single age cohort since fish hatch in synchrony soon after

257 rains fill their natal pools with water (Vrtílek et al. 2018b). The age of fish when sex ratios were

258 estimated was unknown. At each site, fish were collected using a triangular dip net (45 x $45 \mathrm{~cm}$,

259 mesh size $5 \mathrm{~mm}$ ) or beach seine (length $2.7 \mathrm{~m}$, depth $0.7 \mathrm{~m}$, mesh size $4 \mathrm{~mm}$ ). The method retained

260 adult killifish unselectively and there was no sex bias in the probability of capture, confirmed by a

261 combination of capture-mark-recapture studies and removal sampling (Reichard et al. 2014, Vrtílek

262 et al. 2018b). Fish were sorted into species and sexed on the bank, counted and released back to the

263 pool. Details for data collection are provided in Reichard et al. (2014); the new samples used in the

264 present study were collected following an identical protocol. We only used estimates based on at

265 least 6 individuals of a given species in further analyses. Sex ratios were analyzed using a

266 Generalized Linear Mixed Model (GLMM) with binomial error structure (male to female ratio) and

267 log-link function in the Imer package (Pinheiro and Bates 2000), where Species were treated as

268 fixed factor and Year and Site as random factors.

Data on sex ratios at the start of sexual maturity in wild-derived captive populations were

270 collected in captivity from 63 cohorts. Within each cohort, fish were hatched on the same day,

271 following standard husbandry protocol (Polačik et al. 2016). The number of males and females was

272 estimated at age when all fish in the cohort were sexually mature (typically 4-5 weeks). Data were

273 analyzed using a Generalized Linear Model (GLM) with binomial error structure and log-link

274 function. 
Experimental populations. We used fish from four related Nothobranchius species from southern and central Mozambique (Reichard et al. 2017). For laboratory experiments, each species was represented by two independent populations, originating from separate intraspecific lineages (Bartáková et al. 2015). Experimental fish were F1 descendants of wild parents collected in Mozambique. The locations of source populations are presented in Supplementary Table S1. Eggs of parental fish were stored in an incubator (Pollab, Q-CELL $60-240$ ) at $24 \pm 0.5^{\circ} \mathrm{C}$ for at least 16 weeks following standard husbandry protocols (Polačik et al. 2016). The experiment was divided into two phases for logistic reasons (capacity of experimental facility). Work on N. furzeri and $N$. kadleci was completed in September 2011 - December 2012, followed by work on N. orthonotus and N. pienaari (May 2013 - March 2015).

Experimental fish were hatched simultaneously by watering the incubation substrate with dechlorinated tap water $\left(16^{\circ} \mathrm{C}\right)$. From the age of 2-10 days (depending on size of the juveniles, but

288 before the sexes could be separated) fish were housed either in social tanks (24 L) or individually

289 (2L tanks), providing identical fish density between treatments. During the juvenile period, dead

290 experimental fish were replaced with fish of the same age and housing history from stock tanks. At

291 the age of 6-7 weeks, fish in social tanks were marked with a single Visible Implant Elastomer tag

292 (Northwestern Marine Technology) to enable individual recognition, except for $N$. pienaari due to

293 its small size. Previous studies have shown no negative effect of marking on subsequent survival

294 (Sandford et al. 2020). Nine to twelve tanks were used for each study population, with initial

295 density of 12 fish per 24L tank, except for $N$. orthonotus (the largest species) where the density was

29610 fish per tank. Water quality was maintained using air-driven filters and 25-30\% of water was

297 exchanged every 2-3 days. Individually housed fish were kept in 2L tanks in two separate

298 recirculating systems (Aquamedic, Germany, www.aqua-medic.de), with 45 fish per species (22-23 
fish per population). All fish were kept under a $12 \mathrm{~h}: 12 \mathrm{~h}$ light:dark regime in aged tap water

300 (conductivity $550 \mu \mathrm{S} . \mathrm{cm}^{-1}$ ), at a water temperature of $26 \pm 2{ }^{\circ} \mathrm{C}$. Fish were fed twice each day to

301 satiation during the first month and once a day thereafter. Fish were initially fed with live Artemia

302 nauplii and weaned to chopped bloodworm (Chironomus larvae) and Tubifex from the age of 10-30

303 days. All tanks received the same ration (approximately 15\% of body mass of the fish in the tank).

304 Full details are provided in Blažek et al. (2017).

To test the effect of unfavorable environmental conditions arising from fluctuating

temperature (Thomas et al. 1986), we compared sex differences in lifespan in a cohort of

308 (mean \pm SD: $27.5 \pm 1^{\circ} \mathrm{C}$; control fish) or fluctuating temperature (from $20 \pm 1^{\circ} \mathrm{C}$ in early morning to

$30935 \pm 1^{\circ} \mathrm{C}$ in late afternoon). The limits for the fluctuating temperature reflected the diurnal change in

310 water temperature that killifish typically experience in the wild (Žák et al., 2018). A fluctuating

311 temperature was achieved by a combination of an aquarium chiller (TECO TR 10, Italy,

312 www.tecoonline.com $)$ and three aquarium heaters $(2 \times 200 \mathrm{~W}$ and $1 \times 100 \mathrm{~W}$, Eheim/Jäger,

313 Wüstenrot, Germany). Stable temperature in the control group was regulated with one $100 \mathrm{~W}$

314 heater.

316 Lifespan estimates. All tanks were monitored daily for dead fish. Survival was estimated from the

317 age when all fish of a given species were sexually mature (5 weeks in N. orthonotus, 6 weeks in $N$.

318 furzeri and $N$. kadleci, 8 weeks in $N$. pienaari). Sex differences in mortality were analyzed using

319 species-specific Mixed Effects Cox Proportional-Hazards Models (coxme package) (Therneau

320 2015a) with Sex as a fixed factor and Population as a random factor. Note that analysis using

321 Population as the fixed factor (coxph function in survival package, including population by sex 
322 interaction) generated an identical interpretation. Analyses were completed separately for each

323 species and social environment. Fish removed from social tanks for the analysis of functional aging

324 were censored in the survival analysis at the age of removal.

Actuarial aging. Sex-specific mortality hazards were modelled using Bayesian Survival Trajectory

327 Analysis (BaSTA package) (Colchero et al. 2012). First, we used the multibasta command to test

328 whether Gompertz-family models provided a good fit to population-specific survival data. We fitted

329 three basic models (Weibull, Gompertz, Logistic), each with three shape parameters (simple,

330 Makeham, Siler/bathtub) and then compared the fits using Deviance Information Criterion (DIC), a

331 Bayesian equivalent of Akaike Information Criterion. We used 4 runs of each model, each with

332150,000 MCMC iterations, burn in of 15,000 and thinning by sampling every 50th estimate. We

333 analyzed each population separately as we knew a priori that populations within species differ in

334 lifespan (Blažek et al. 2017) and, unlike for survival analysis, they cannot be entered as random

335 effects to BaSTA. Gompertz-family models were chosen to provide the most unambiguous

336 demographic interpretation of the parameters (Bronikowski et al. 2011; Boonekamp et al. 2020) and

337 a good fit to the datasets. The Gompertz model assumes that aging starts at species-specific age,

338 with one parameter (intercept, Initial mortality rate, IMR) describing age-independent mortality

339 (baseline mortality) and the second parameter (slope, Rate of Aging, RoA) describing the increase in

340 mortality with age (Pletcher et al. 2000). In both $N$. furzeri populations, deceleration in aging was

341 apparent at old age, probably arising from intra-population heterogeneity (Chen et al. 2013), and

342 Gompertz-logistic models were used as their DIC was considerably lower than a simple Gompertz

343 model. A Gompertz-logistic model estimates a third parameter $(s)$ which models deceleration of

344 aging rate at old age. The final models were run with 400,000 MCMC iterations, burn in of 50,000 
345 and thinning by sampling every 50th estimate to provide a posterior distribution of parameters for

346 each species. Model parameters were compared between males and females using Kullback-Leibler

347 discrepancy criterion (KL). The KL varies between 0.5 (complete overlap) to 1.0 (no overlap) with

348 the values $>0.8$ are considered as a substantial difference (Colchero et al. 2012).

350 Oxidative stress. Subsamples of 20 young and old fish from social treatment (young fish: 12

351 weeks; old fish: 26 weeks in N.furzeri and N. kadleci and 30 weeks in N. orthonotus and N.

352 pienaari), were sacrificed for per species (equal representation of sex and populations). Their brain,

353 liver and heart were flash frozen in liquid nitrogen. Tissues were homogenized in acetonitrile with

354 deuterium-labelled internal standards, and oxidation products of nucleic acids (8-hydroxy-2'-

355 deoxyguanosine (8-OHdG); 8-hydroxyguanosine (8-OHG)), proteins (o-Tyrosine, 3-nitrotyrosine,

356 3-chlorotyrosine) and lipids (8-isoprostane) were determined by liquid chromatography-

357 electrospray-high resolution mass spectrometry (HPLC-ESI-HRMS) as described in Blažek et al.

358 (2017). N. orthonotus and $N$. pienaari from the main experiment were used, but new cohorts of $N$.

359 furzeri and N. kadleci were raised using identical conditions (Blažek et al. 2017). Within

360 individuals, data from the three organs and biomarkers were collinear. We combined data across

361 organs and biomarkers using Principal Component Analysis (Supplementary Table S3). The first

362 PC explained $71.8 \%$ of variability and had positive loadings with all biomarkers across all tissues

363 (0.77-0.92). We used a Linear Mixed Model to test how the sexes differed in oxidative stress (using

$364 P C 1$ as a fixed effect) and how increase in oxidative stress with age varied between the sexes (i.e. a

365 Sex by Age interaction). Population ID was a random effect. In addition to an overall test of

366 oxidative stress, we used organ-specific and biomarker-specific analyses (Supplementary Tables S4

367 and S5), which were fully concordant with the PC1-based analysis. 
369 Histopathology. Another sample of young (age 14 weeks) and old (age 23 weeks) N. furzeri and $N$.

370 kadleci from social treatment was sacrificed (20 fish per age and species). Liver and kidney were

371 preserved in Baker's solution, embedded in Paraplast, sectioned (5 $\mu \mathrm{m})$ and stained in H\&E. From

372 the histological slides, the incidence of proliferative changes was scored using a 5-grade

373 pathological scale (Di Cicco et al. 2011) (score 0-4, 0: no proliferation, $4:>50 \%$ of tissue filled with

374 proliferative cells). Data were analyzed using Cumulative Link Mixed models for ordinal data in the

375 package ordinal (Christensen, 2019), with Sex, Age and their interaction as fixed factors, and

376 Population ID as a random factor. The amount of lipofuscin particles was estimated from separate

377 slides (unstained sections) using a Leica confocal fluorescent microscope. Nine slides were

378 analyzed for each individual. Excitation wavelength was set to $488 \mathrm{~nm}$ (confocal parameters as

379 pinhole, photo-multiplier and laser intensity were fixed). Images were imported to imageJ and the

380 number of lipofuscin (fluorescing) particles counted. Data were analyzed using Generalized Linear

381 Mixed models with Poisson errors (counts), with Sex, Age and their interaction as fixed factors, and

382 Population ID and Individual ID as random effects.

384 Data availability. All data associated with paper are available in the Figshare repository (doi:

385 10.6084/m9.figshare.12752648).

387 ACKNOWLEDGEMENTS

388 Funding came from Czech Science Foundation (19-01781S) to M.R. All work was carried out in 389 accordance with relevant guidelines and regulations. Fieldwork complied with legal regulations of 390 Mozambique (collection licenses: DPPM/053/7.10/08, 175/154/ IIP/2009/DARPE, 
392 work was approved by the Ethical Committee of the Institute of Vertebrate Biology (No. 163-12)

393 and by Ministry of Agriculture (CZ 62760203) in accordance with legal regulations of the Czech

394 Republic. We thank C. Smith for valuable comments.

\section{AUTHOR CONTRIBUTIONS}

397 MR conceived and designed the study, conducted statistical analyses and drafted the manuscript.

398 MR, RB and MP collected data on wild populations. RB completed the experiment with captive

399 fish, with the assistance of MP. JZ collected data from fluctuating temperature. PK analyzed tissues

400 for oxidative stress. OT designed and interpreted oxidative stress data. TA established and managed

401 team for oxidative stress analysis. AC and RB performed histological analysis. All authors

402 contributed to the final text. 


\section{REFERENCES}

Arnold SJ. 1994. Bateman's principles and the measurement of sexual selection in plants and animals. American Naturalist 144:S1-S22. DOI: 10.1086/285656

Austad SN, Fischer KE. 2016. Sex differences in lifespan. Cell Metabolism 23:1022-1033. DOI: 10.1016/j.cmet.2016.05.019

Austad SN. 2006. Why women live longer than men: sex differences in longevity. Gender Medicine 3:7992. DOI: $10.1016 / \mathrm{S} 1550-8579(06) 80198-1$

Bartáková V, Reichard M, Blažek R, Polačik M, Bryja J. 2015. Terrestrial fishes: rivers are barriers to gene flow in annual fishes from the African savanna. Journal of Biogeography 42:1832-1844. DOI: $10.1111 /$ jbi.12567

Bates D, Maechler M, Bolker B, Walker S. 2014. Lme4: Linear mixed-effects models using Eigen and S4. R package v. 1.1-6. Available at: https://cran.r-project.org/web/packages/lme4/lme4.pdf. Accessed: 6 April 2020

Baumgart M, Di Cicco E, Rossi G, Cellerino A, Tozzini ET. 2015. Comparison of captive lifespan, ageassociated liver neoplasias and age-dependent gene expression between two annual fish species: Nothobranchius furzeri and Nothobranchius korthausae. Biogerontology 16:63-69. DOI: 10.1007/s10522-014-9535-y

Beirne C, Delahay R, Young A.2015. Sex differences in senescence: the role of intra-sexual competition in early adulthood. Proceedings of the Royal Society B: Biological Sciences 282:20151086. DOI: 10.1098/rspb.2015.1086

Blažek R, Polačik M, Kačer P, Cellerino A, Řežucha R, Methling C, Tomášek O, Syslová K, TerzibasiTozzini E, Albrecht T, Vrtílek M, Reichard M. 2017. Repeated intra-specific divergence in lifespan and ageing of African annual fishes along an aridity gradient. Evolution 71:386-402 (2017). DOI: 10.1111/evo.13127

Bonduriansky R, Maklakov A, Zajitschek F, Brooks R. 2008. Sexual selection, sexual conflict and the evolution of ageing and life span. Functional Ecology 22:443-453. DOI: 10.1111/j.13652435.2008.01417.x

Boonekamp JJ, Bauch C, Verhulst S. 2020. Experimentally increased brood size accelerates actuarial senescence and increases subsequent reproductive effort in a wild bird population. Journal of Animal Ecology 89:1395-1407. DOI: 10.1111/1365-2656.13186

Bronikowski AM, Altmann J, Brockman DK, Cords M, Fedigan LM, Pusey A, Stoinski T, Morris WF, Strier KB, Alberts SC. 2011. Aging in the natural world: comparative data reveal similar mortality patterns across primates. Science 331:1325-1328 (2011). DOI: 10.1126/science.1201571

Cellerino A, Valenzano DR, Reichard M. 2016. From the bush to the bench: the annual Nothobranchius fishes as a new model system in biology. Biological Reviews 91:511-533. DOI: 10.1111/brv.12183

Chen HY, Zajitschek F, Maklakov AA. 2013. Why ageing stops: heterogeneity explains late-life mortality deceleration in nematodes. Biology Letters 9:20130217. DOI: 10.1098/rsbl.2013.0217

Christensen RHB. 2019. Ordinal - Regression Models for Ordinal Data. R package version 2019.12-10. Available at: https://CRAN.R-project.org/package=ordinal. Accessed: 6 April 2020

Clutton-Brock TH, Isvaran K. 2007. Sex differences in ageing in natural populations of vertebrates. Proceedings of the Royal Society B: Biological Sciences 274:3097-104. DOI: 10.1098/rspb.2007.1138 
Colchero F, Jones OR, Rebke M. 2012. BaSTA: an R package for Bayesian estimation of age-specific survival from incomplete mark-recapture/recovery data with covariates. Methods in Ecology and Evolution 3:466-470. DOI: 10.1111/j.2041-210X.2012.00186.x

Cui R, Medeiros T, Willemsen D, Iasi LNM, Collier GE, Graef M, Reichard M, Valenzano DR. 2019. Relaxed selection limits lifespan by increasing mutation load. Cell 178:385-399. DOI: 10.1016/j.cell.2019.06.004

Di Cicco E, Tozzini ET, Rossi G, Cellerino A. 2011. The short-lived annual fish Nothobranchius furzeri shows a typical teleost aging process reinforced by high incidence of age-dependent neoplasias. Experimental Gerontology 46:249-256. DOI: 10.1016/j.exger.2010.10.011

Fisher RA. 1930. The Genetical Theory of Natural Selection. (Oxford University Press, Oxford).

Foo YZ, Nakagawa S, Rhodes G, Simmons LW. 2017. The effects of sex hormones on immune function: a meta-analysis. Biological Reviews 92, 551-571. DOI: 10.1111/brv.12243

Frank SA, Hurst LD. 1996. Mitochondria and male disease. Nature 383:224-224. DOI: 10.1038/383224a0

Gemmell NJ, Metcalf VJ, Allendorf FW. 2004. Mother's curse: the effect of mtDNA on individual fitness and population viability. Trends in Ecology \& Evolution 19:238-244.

Genade T. 2005. Laboratory manual for culturing N. furzeri. Available at: http://www.nothobranchius.info/pdfs/lab_protocols_1.pdf. Accessed: 25 August 2020

Gordon EH, Peel NM, Samanta M, Theou O, Howlett SE, Hubbard RE. 2017. Sex differences in frailty: a systematic review and meta-analysis. Experimental Gerontology 89:30-40. DOI: 10.1016/j.exger.2016.12.021

Gotthard K, Nylin S, Wiklund C. 2000. Mating opportunity and the evolution of sex-specific mortality rates in a butterfly. Oecologia 122:36-43. DOI: 10.1007/PL00008833

Gupta S, Nakabo S, Blanco LP, O’Neil LJ, Wigerblad G, Goel RR, Mistry P, Jiang K, Carmona-Rivera C, Chan DW, Wang X, Pedersen HL, Gadkari M, Howe KN, NAz F, Dell'Orso S, Hasni SA, Dempsey C, Buscetta A, Frischmeyer-Guerrerio PA, Kruszka P, Muenke M, franco ML, Sun HW, Kaplan MJ. 2020. Sex differences in neutrophil biology modulate response to type I interferons and immunometabolism. Proceedings of the National Academy of Sciences of the United States of America 117:16481-16491. DOI: 10.1073 /pnas.2003603117

Haas R. 1976. Sexual selection in Nothobranchius guentheri (Pisces: Cyprinodontidae). Evolution 30:614622. DOI: $10.1111 / \mathrm{j} .1558-5646.1976 . t b 00938 . x$

Hu CK, Brunet A. 2018. The African turquoise killifish: A research organism to study vertebrate aging and diapause. Aging Cell 17:e12757. DOI: 10.1111/acel.12757

Jung T, Bader N, Grune T. 2007. Lipofuscin: formation, distribution, and metabolic consequences. Annals of the New York Academy of Sciences 1119:97-111. DOI: 10.1196/annals.1404.008

Keller S, Weiss J, Schleifer S, Miller N, Stein M. 1992. Suppression of immunity by stress: effect of a graded series of stressors on lymphocyte stimulation in the rat. Science 213:1397-1400. DOI: $10.1126 /$ science. 6973822

Keppeler FW, Cruz DA, Dalponti G, Mormul RP. 2016. The role of deterministic factors and stochasticity on the trophic interactions between birds and fish in temporary floodplain ponds. Hydrobiologia 773:225-240. DOI: 10.1007/s10750-016-2705-y

Krysanov E, Demidova T. 2018. Extensive karyotype variability of African fish genus Nothobranchius (Cyprinodontiformes). Comparative Cytogenetics 12:387. DOI: 10.3897/CompCytogen.v12i3.25092 
Lemaître JF, Ronget V, Tidière M, Allainé D, Berger V, Cohas A, Colchero F, Conde DA, Garrat M, iker A, Marais GAB, Sheuerlein A, Székely T, Gaillard JM. 2020. Sex differences in adult lifespan and aging rates of mortality across wild mammals. Proceedings of the National Academy of Sciences of the United States of America 117:8546-8553. DOI: 10.1073/pnas.1911999117

Liker A, Székely T. 2005. Mortality costs of sexual selection and parental care in natural populations of birds. Evolution 59:890-7. DOI: 10.1111/j.0014-3820.2005.tb01762.x

Maklakov AA, Lummaa V. 2013. Evolution of sex differences in lifespan and aging: causes and constraints. BioEssays 35:717-724. DOI: 10.1002/bies.201300021

Moore SL, Wilson K. 2002. Parasites as a viability cost of sexual selection in natural populations of mammals. Science 297:2015-8. DOI: 10.1126/science.1074196

Pletcher SD, Khazaeli AA, Curtsinger JW. 2000. Why do life spans differ? Partitioning mean longevity differences in terms of age-specific mortality parameters. The Journals of Gerontology: Series A 55:B381-B389. DOI: 10.1093/gerona/55.8.B381

Polačik M, Blažek R, Reichard M. 2016. Laboratory breeding of the short-lived annual killifish Nothobranchius furzeri. Nature Protocols 11:1396. DOI: 10.1038/nprot.2016.080

Polačik M, Reichard M. 2011. Asymmetric reproductive isolation between two sympatric annual killifish with extremely short lifespans. PLoS One 6:e22684. DOI: 10.1371/journal.pone.0022684

Promislow D. 2003. Mate choice, sexual conflict, and evolution of senescence. Behavior Genetics 33:191201. DOI: $10.1023 /$ A:1022562103669

Promislow DE, Montgomerie R, Martin TE. 1992. Mortality costs of sexual dimorphism in birds. Proceedings of the National Academy of Sciences of the United States of America 250:143-150.

Regan JC, Partridge L. 2013. Gender and longevity: why do men die earlier than women? Comparative and experimental evidence. Best Practice \& Research: Clinical Gastroenterology 27:467-479. DOI: 10.1016/j.beem.2013.05.016

Reichard M, Polačik M. 2019. Nothobranchius furzeri, an 'instant' fish from an ephemeral habitat. eLife 8:e41548. DOI: $10.7554 /$ eLife.41548.001

Reichard M, Polačik M, Blažek R, Vrtílek M. 2014. Female bias in the adult sex ratio of African annual fishes: interspecific differences, seasonal trends and environmental predictors. Evolutionary Ecology 28:1105-1120. DOI: $10.1007 / \mathrm{s} 10682-014-9732-9$

Sandford M, Castillo G, Hung TC. 2020. A review of fish identification methods applied on small fish. Reviews in Aquaculture 12:542-554. DOI: 10.1111/raq.12339

Sedláček O, Baciaková B, Kratochvíl L. 2014. Evolution of body colouration in killifishes (Cyprinodontiformes: Aplocheilidae, Nothobranchiidae, Rivulidae): Is male ornamentation constrained by intersexual genetic correlation? Zoologischer Anzeiger - A Journal of Comparative Zoology 253:207215. DOI: $10.1016 /$ j.jcz.2013.12.004

Sielezniew M, Kostro-Amboziak K, Korosi Á. 2020. Sexual differences in age-dependent survival and life span of adults in a natural butterfly population. Scientific Reports 10:10394. DOI: 10.1038/s41598-02066922-w

Sowersby W, Gonzalez-Voyer A, Rogell B. 2020. Sex ratios deviate across killifish species without clear links to life history. Evolutionary Ecology 34:411-426. DOI: 10.1007/s10682-020-10041-5 
Szekely T, Liker A, Freckleton RP, Fichtel C, Kappeler PM. 2014. Sex-biased survival predicts adult sex ratio variation in wild birds. Proceedings of the Royal Society B: Biological Sciences 281:20140342. DOI: $10.1098 / \mathrm{rspb} .2014 .0342$

Terzibasi-Tozzini E, Dorn A, Ng’oma E, Polačik M, Blažek R, Reichwald K, Petzold A, Watters B, Reichard M, Cellerino A. 2013. Parallel evolution of senescence in annual fishes in response to extrinsic mortality. BMC Evolutionary Biology 13:1-12. DOI: 10.1186/1471-2148-13-77

Therneau TM. 2015. Mixed effects Cox models, R package version 2.2-5. Available at: https://cran.rproject.org/web/packages/coxme/coxme.pdf. Accessed: 6 April 2020

Thomas RE, Gharrett JA, Carls MG, Rice SD, Moles A, Korn S. et al., Effects of fluctuating temperature on mortality, stress, and energy reserves of juvenile coho salmon. Transactions of the American Fisheries Society 115:52-59. DOI: 10.1577/1548-8659(1986)115<52:EOFTOM>2.0.CO;2

Tompkins EM, Anderson DJ. 2019. Sex-specific patterns of senescence in Nazca boobies linked to mating system. Journal of Animal Ecology 88:986-1000. DOI: 10.1111/1365-2656.12944

Trivers R. 1972. Parental investment and sexual selection. In: Campbell B (ed.). Sexual Selection and the Descent of Man 1871-1971, Aldine, pp. 136-179.

Tuttle MD, Ryan MJ. 1981. Bat predation and the evolution of frog vocalizations in the Neotropics. Science 214:677-678. DOI: 10.1126/science.214.4521.677

Vrtílek M, Žák J, Polačik M, Blažek R, Reichard M. 2018. Longitudinal demographic study of wild populations of African annual killifish. Scientific Reports 8:1-12. DOI: 10.1038/s41598-018-22878-6

Vrtílek M, Žák J, Pšenička M, Reichard M. 2018. Extremely rapid maturation of a wild African annual fish. Current Biology 28:R822-R824. DOI: 10.1016/j.cub.2018.06.031

Wildekamp RH. 2004. A world of killies: atlas of the oviparous cyprinodontiform fishes of the world, Vol. 4. American Killifish Association, Elyria.

Xirocostas ZA, Everingham SE, Moles AT. 2020. The sex with the reduced sex chromosome dies earlier: a comparison across the tree of life. Biology Letters 16:20190867. DOI: 10.1098/rsbl.2019.0867

Žák J, Reichard M, Gvoždík L. 2018. Limited differentiation of fundamental thermal niches within the killifish assemblage from shallow temporary waters. Journal of Thermal Biology 78:257-262. DOI: 10.1016/j.jtherbio.2018.10.015 


\section{$556 \quad$ Figure legends}

557 Fig. 1. Proportion of males at the onset of sexual maturity in wild-derived laboratory populations

558 (grey columns) and in wild populations (green columns). Means with 95\% confidence intervals,

559 back-calculated from outcomes of binomial models, are shown.

561 Fig. 2. Sex-specific posterior distribution of baseline mortality (IMR), rate of aging (RoA),

562 survival, and mortality risk estimated from Gompertz model for both $N$. orthonotus populations (A,

563 B) and both $N$. furzeri populations (C, D) in the social treatment. In N. furzeri, the posterior

564 distribution for aging deceleration parameter $(s)$ is also presented. KL denotes Kullback-Leibler

565 discrepancy criterion, with values $>0.8$ considered as a substantial difference. 


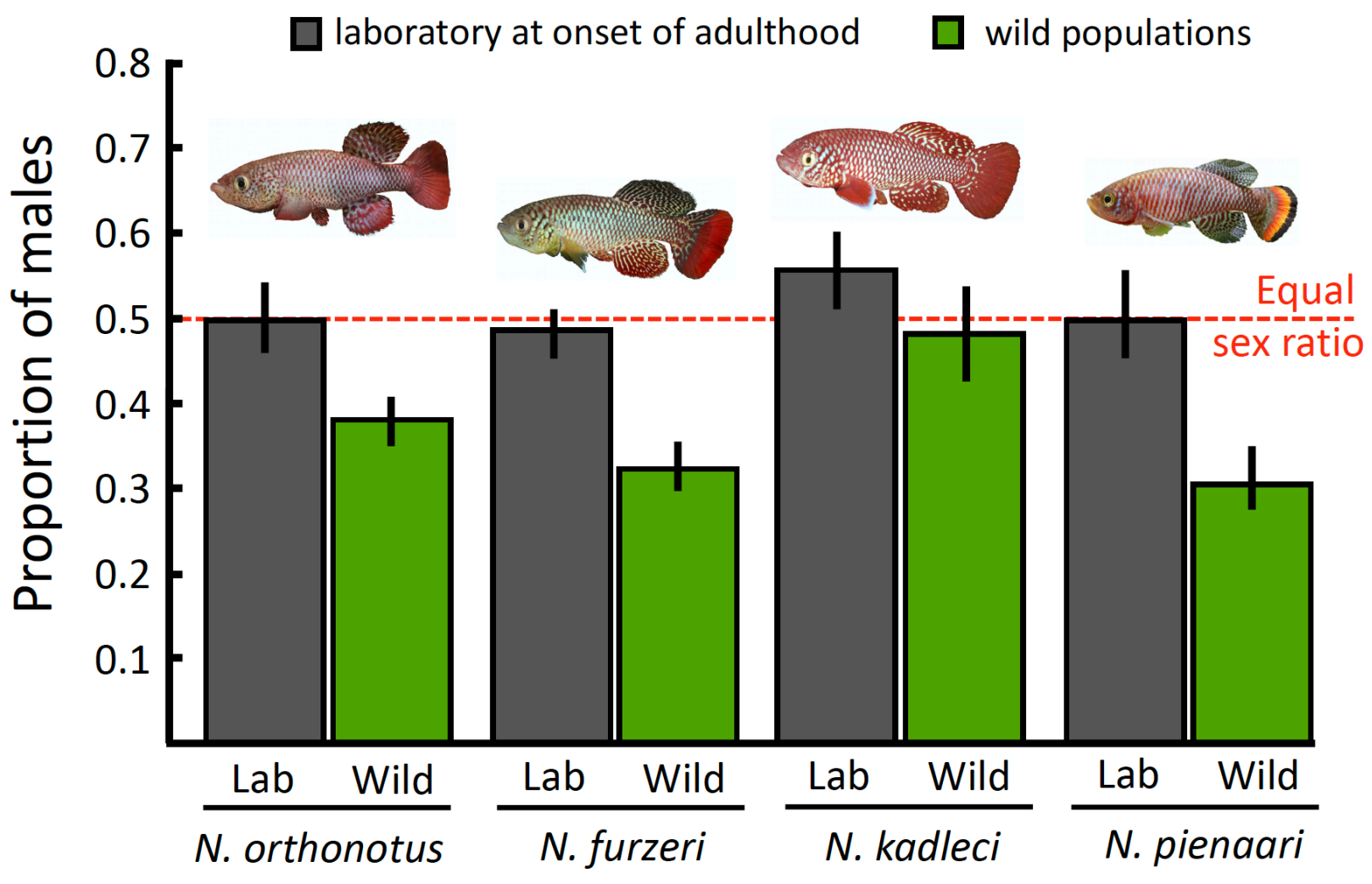


Fig. 2

\section{A. N. orthonotus}
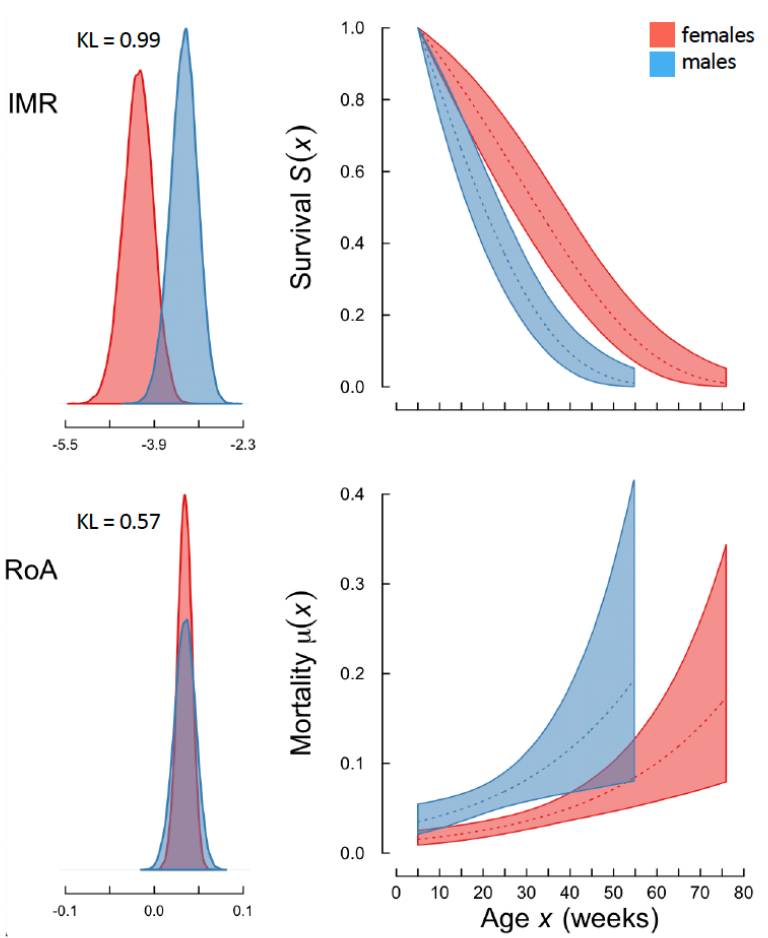

C. N. furzeri
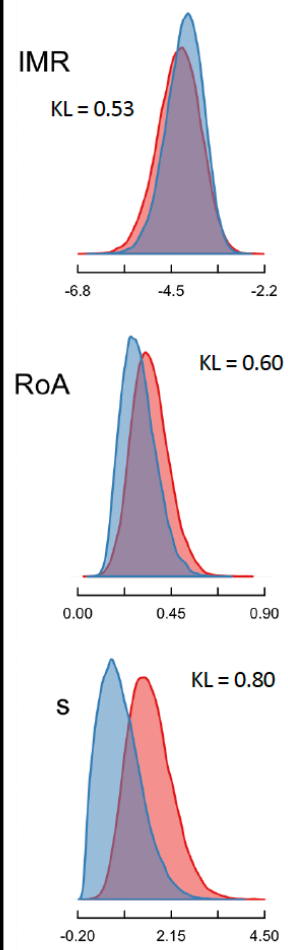

B. N. orthonotus
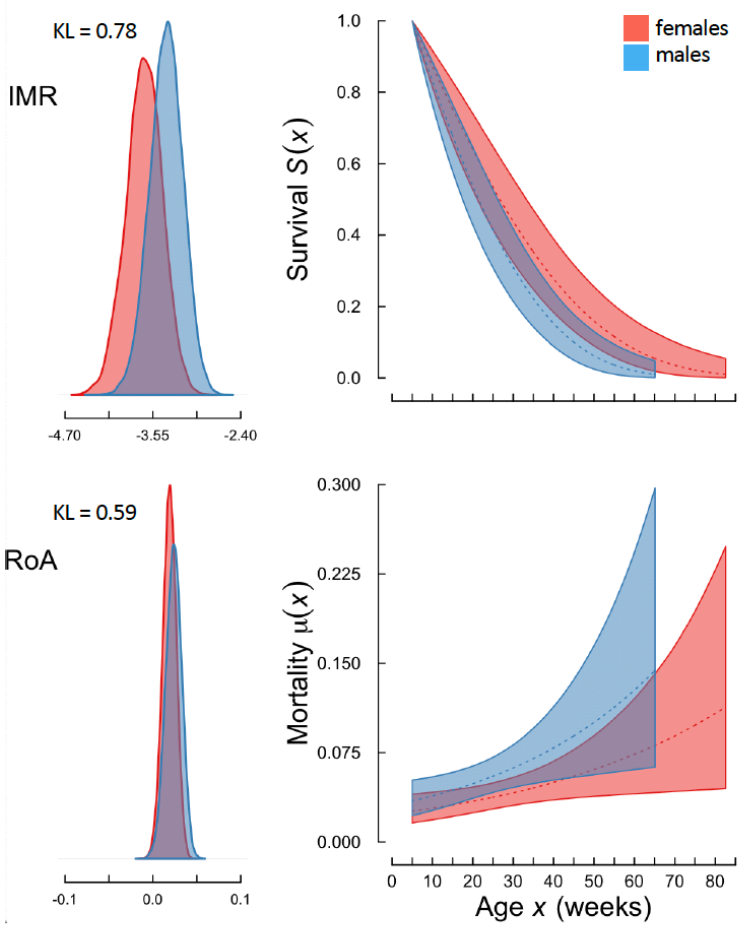

D. N. furzeri
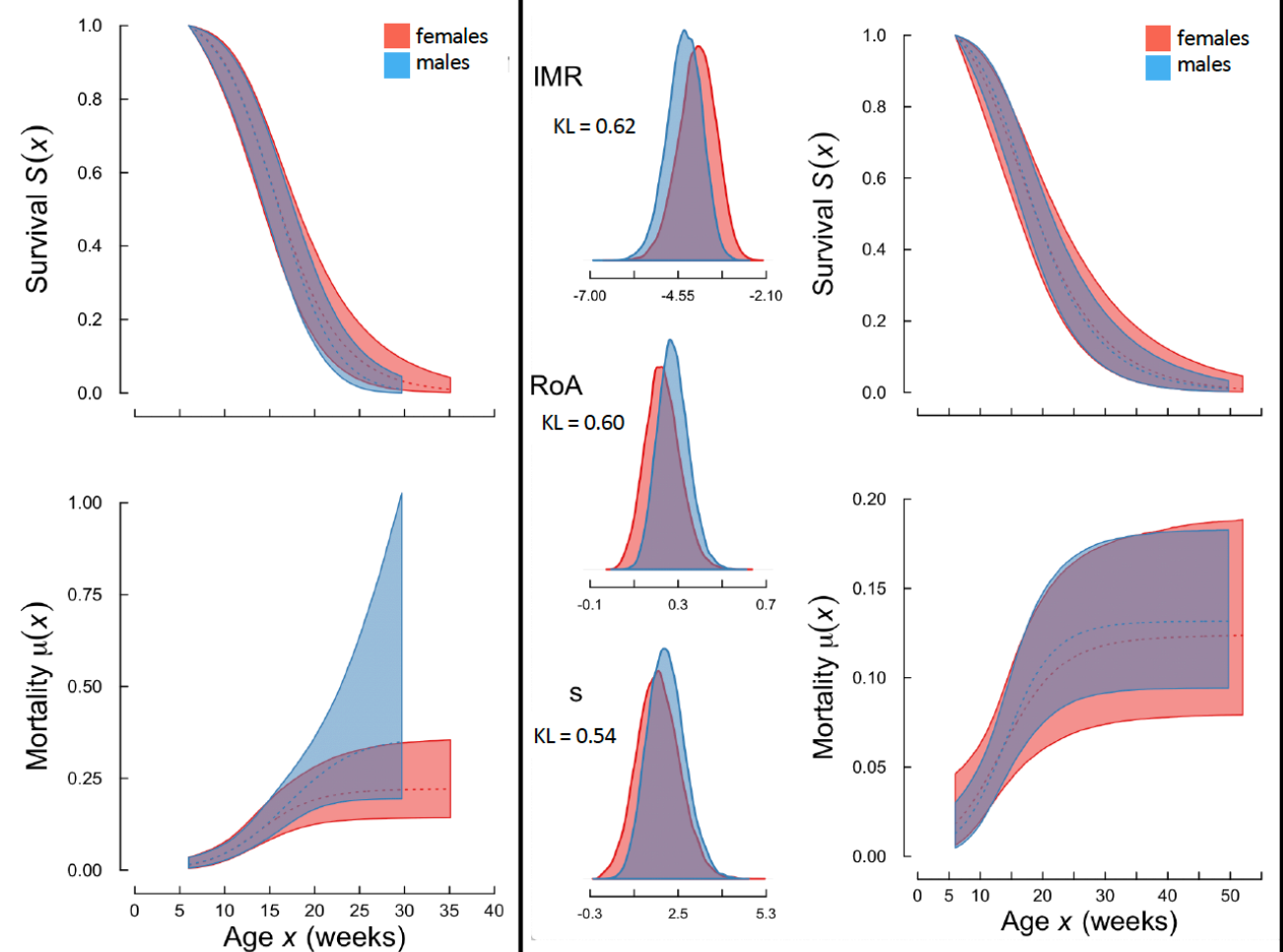
572 Table 1. Sex-specific median lifespan estimates (with 95\% confidence intervals estimated from the

573 survfit function) in the social and single housing treatments. NAs represent cases where the upper

574 confidence interval cannot be reliably calculated.

575

\begin{tabular}{llll}
\hline & & \multicolumn{2}{l}{ Median lifespan (95\% CI) } \\
\hline Species & Sex & Social & Single \\
\hline N. orthonotus & Females & $256(214-289)$ & $232(193-352)$ \\
& Males & $180(134-197)$ & $271(215-368)$ \\
N.furzeri & Females & $150(131-176)$ & $121(104-\mathrm{NA})$ \\
& Males & $121(115-134)$ & $117(111-145)$ \\
N. kadleci & Females & $172(148-272)$ & $166(103-240)$ \\
& Males & $178(161-206)$ & $113(97-\mathrm{NA})$ \\
& Females & $282(221-338)$ & $314(242-347)$ \\
& Males & $251(227-286)$ & $297(235-446)$ \\
\hline
\end{tabular}


577 Table 2. The effects of sex, age and their interaction on proliferative changes to liver (a), deposition

578 of lipofuscin (b) and proliferative changes to kidney (c). Parameter estimates and z-statistics of

579 Generalized Mixed Models are presented.

580

\begin{tabular}{llclcr}
\hline Organ impairment & Factor & Estimate & \pm s.e. & z-value & P \\
\hline Proliferative changes - liver & Intercept & 0.761 & \pm 0.242 & 3.14 & 0.002 \\
& Sex(males) & -0.110 & \pm 0.218 & -0.50 & 0.615 \\
& Age(young) & -0.240 & \pm 0.225 & 1.07 & 0.287 \\
& Sex x Age & 0.449 & \pm 0.310 & 1.45 & 0.147 \\
& & & & & \\
\hline Lipofuscin - liver & Intercept & 3.099 & \pm 0.263 & 11.78 & $<0.001$ \\
& Sex(males) & -0.045 & \pm 0.259 & -0.17 & 0.862 \\
& Age(young) & 0.071 & \pm 0.045 & 1.57 & 0.115 \\
& Sex x Age & -0.195 & \pm 0.299 & -0.65 & 0.514 \\
& & & & & \\
\hline Proliferative changes - kidney & Intercept & 0.338 & \pm 0.347 & 0.97 & 0.331 \\
& Sex(males) & -0.481 & \pm 0.281 & -1.71 & 0.087 \\
& Age(young) & -0.637 & \pm 0.353 & -1.81 & 0.071 \\
& Sex x Age & 0.551 & \pm 0.474 & 1.16 & 0.247
\end{tabular}

